\title{
A STUDY ON OPTICAL CHARACTER RECOGNITION TECHNIQUES
}

\author{
Narendra Sahu ${ }^{1}$ and Manoj Sonkusare ${ }^{2}$ \\ ${ }^{1}$ Department of Computer Sc. \& Engg., Women's Polytechnic College, Indore, India \\ ${ }^{2}$ Department of Computer Sc. \& Engg., Ujjain Engineering College, Ujjain, India
}

\begin{abstract}
:
Optical Character Recognition (OCR) is the process which enables a system to without human intervention identifies the scripts or alphabets written into the users' verbal communication. Optical Character identification has grown to be individual of the mainly flourishing applications of knowledge in the field of pattern detection and artificial intelligence. In our survey we study on the various OCR techniques. In this paper we resolve and examine the hypothetical and numerical models of Optical Character Identification. The Optical character identification or classification (OCR) and Magnetic Character Recognition (MCR) techniques are generally utilized for the recognition of patterns or alphabets. In general the alphabets are in the variety of pixel pictures and it could be either handwritten or stamped, of any series, shape or direction etc. Alternatively in MCR the alphabets are stamped with magnetic ink and the studying machine categorize the alphabet on the basis of the exclusive magnetic field that is shaped by every alphabet. Both MCR and OCR discover utilization in banking and different trade appliances. Earlier exploration going on Optical Character detection or recognition has shown that the In Handwritten text there is no limitation lying on the script technique. Hand written correspondence is complicated to be familiar through due to diverse human handwriting style, disparity in angle, size and shape of calligraphy. An assortment of approaches of Optical Character Identification is discussed here all along through their achievement.
\end{abstract}

\section{KEYWORDS:}

Optical Character Identification, offline Hand written Character Recognition, Pre-processing, characteristic Extraction, categorization, Post Processing.

\section{INTRODUCTION}

Optical Character Identification is single of the majority enthralling and demanding areas of pattern recognition with a variety of realistic applications. The times departed by allow distinguish us that OCR expertise has been built by lots of researchers over a long period of time, consisting unreservedly of impressive like a worldwide human research network. In such an imperceptible discussion, people have made efforts, with "antagonism and collaboration," to advance the research effort. In this way, global symposiums and inductions are being determined to stimulate the improvement in the domain. For example the global induction on Frontiers in Handwriting detection and the International discussion on article psychoanalysis and Recognition determination play an explanation task in the intellectual and matter-of-fact arena [2].

All neural networks want data to be skilled. These data consist of input and target data. The guidance of one alphabet could be done with an input and an output vector. The length of the input vector will be the resolution of the input. I.e. if they worked with the fourth network, then the length will be 77 elements, every row coming after each other in it. The elements could have values 0 or 1 . If the grid part of the image represented by the vector constituent has more than $50 \%$ coverage of the letter's features, after that the vector element's value is 1 , otherwise it is 0 . 
The International Journal of Computational Science, Information Technology and Control Engineering

(IJCSITCE) Vol.4, No.1, January 2017

By checking this for all of the grid parts, we get the vector. During the primary neural network we have 10 numbers. This funds we will have 10 rows of this kind of vectors, every row representing a number. This describes the input matrix. This kind of data form is expected as inputs through Matlab in the neural network. For a network with $\mathrm{N}$ outputs, the output matrix will be an NxN dimension matrix, filled with zeros, and having ones on the main diagonal. This step of the recognition was done manually [6].

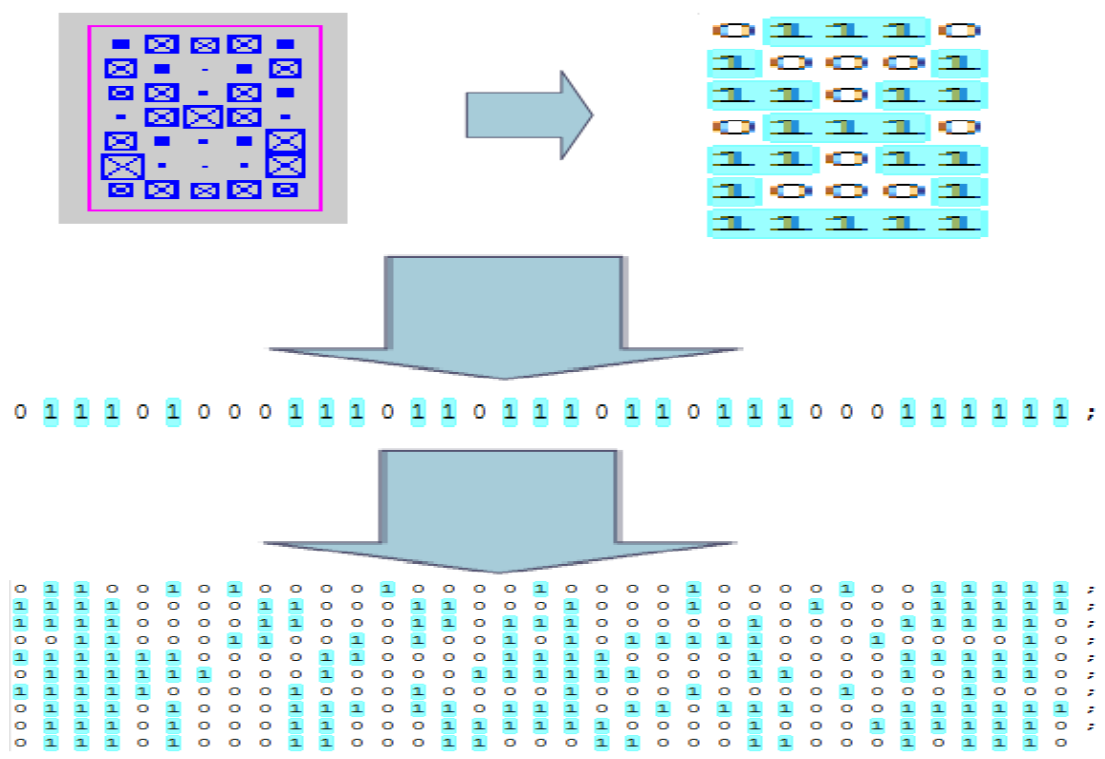

Figure.1. the look of the input data

Until a few decades previously, explore within the domain of Optical Character identification was restricted to document images acquired with flatbed desktop scanners [4]. The efficiency of such methods is incomplete as they aren't exchangeable because of huge dimension of the digital scanners and the requirement of a computing method. Furthermore, the capturing speed of a digital scanner is dull than that of a high resolution digital camera. Recently, with the development of dispensation velocity and interior recollection of mobile devices such as high-end cell-phones, Individual Digital Assistants (IDA), smart phones, iPhones, iPods, etc. having builtin digital cameras, an innovative trend of explore has emerged into representation. Researchers have dared to imagine of organization OCR applications on such strategy for having authentic instant outcome. A habitual big industry credential Reader (ICR), destined for repeated inhabitants of relevant contact in sequence starting a trade card also recognized as visiting card into the phone book of the diplomacy is an example of such applications. Conversely, computing in hand held approach engages a technique to of challenge. Since of the non contact environment of digital cameras connected to hand held machines, obtained descriptions terribly often undergo from skew and perception bend.

\section{Optical Character Recognition}

During accumulation to that, manual association in the capturing procedure, irregular and inadequate explanation, and unavailability of difficult focusing system yield poor quality images. The dealing out velocity and recollection size of handheld devices are not up till now sufficient enough so as to run desktop based OCR algorithms that are computationally luxurious and necessitate elevated quantity of recollection. In addition to the challenges in recognition of 
characters, it is proficient to contribute greatly to the progression of a mechanization procedure along with could improve the interface connecting man and machine.

It is the method to renovate mechanism in print, dispense in print or hand printed article interested in editable manuscript arrangement. Typically, there are two dissimilar categories of handwriting character detection: off-queue and on-queue. Inside Online character identification script is captured by an unusual highlighter during concurrence through electronic peripheral. Offline character detection participation have been scanned as of a plane such as expanse of document and stored digitally. Offline character identification enclose detection of mechanism in print, hand printed and handwritten typeset. The majority complicated difficulty in the field of OCR is the identification of unimpeded cursive script. Place for the duration of the eighties. In nineties, an unmarked curiosity urbanized by means of the increase new requirements. The obtainable equipment for modeling is not until now enough by admiration to presentation appropriate to a lot of variation of creature script. Similarity in individual quality shapes, the overlaps, and interconnection of the neigh boring alphabets auxiliary make difficult the predicament.

Alphabet appreciation systems offer potential advantages by provided that an interface that facilitates interfaces connecting man and mechanism. Some of the application areas where OCR acting a extremely significant character consist of archiving documents, automatic substantiation of checks, data entry and wide variety of business-oriented applications [14]. During the precedent few years' precious research has been done in the domain of alphabet recognition, and a huge amount of research manuscripts and news were dedicated on this subject. Pre-Processing be able to be distinct as maintenance the manuscript representation and creation It apposite for Input to the OCR Engine. Most significant Steps under Pre-Processing Are:

- Noise elimination

- Skew recognition/improvement

- Binarization

The sound introduces through the visual scan strategy within the effort lead to unfortunate scheme performance. These imperfections must be detached earlier to spirit detection. Sound is able to be introducing in an illustration through image acquirement along with communication. Sound could be of poles separately type as Gaussian sound, Gamma sound, Rayleigh sound, Exponential sound, Uniform sound, salty and sprinkle sound, intermittent sound etc. Sound could be impassive by model filter, Butterworth filter and Gaussian filter. Readily available is an opportunity of revolution of illustration as scan. Skew recognition and modification is old to line up the document with the synchronize scheme of scanner. A variety of skew recognition technique is projection profile, associated mechanism, Hough transform, cluster etc. Binarization, shade or grey-level illustration is transformed addicted to twofold picture with the help of threshold. Binary image could be achieved using Adaptive threshold, Global threshold, variable threshold, method etc. Morphological operations are also utilized in pre-processing. Dilation and Erosion are the morphological operations that amplify or diminish the image size. Erosion makes an entity slighter next to erode away the pixels as of its ends. All entity pixels that are touching backdrop pixels are altered into backdrop pixel. However, dilation creates an article generously proportioned by accumulation pixels approximately its ends. All pixels that are touching an entity pixel are altered into object pixel. Other morphological operations are opening and closing.

The entire identification method of optical character is calculated to covenant with bi-tonal pictures of black passage on white environment. Yet in this outwardly plain imaging territory, there subsist a range of picture configures because of distinctions in picture spatial colour, programming approaches, resolutions and compression formats. Usual reproduction scanned 
The International Journal of Computational Science, Information Technology and Control Engineering (IJCSITCE) Vol.4, No.1, January 2017

document images are bi-tonal (solitary bit for each pixel) with spatial resolutions of 200 dots for every inch (dpi). This resolution may not always be sufficient for high-quality OCR. Contemporary character recognition engines work improved with documents scanned at $300 \mathrm{dpi}$ or higher. Online libraries seeking to practically defend the original article superiority that frequently want gray scale or smooth precise-color is inspect at $600 \mathrm{dpi}$.

High-resolution colour images, however, take their toll on a system's storage, and could become prohibitively large to store in large quantities. Smart digital libraries utilized different compression schemes for text and non- text content. A general compression occupied for bi-tonal manuscript pictures is CCITT Fax Group 3 and Group 4 [16], whereas actual humankind imagery is greatest condensed with JPEG method. Below we characterize some of the most well-liked representation and organizer format and their applicability to OCR.

TIFF stands for Tagged Image File Format. It is solitary of the majorities fashionable and supple of the present communal field raster file formats considered for raster data interchange. TIFF was developed mutually by Aldus and Microsoft Corporation. Adobe Systems acquired Aldus in addition to at the present holds the patent for the TIFF requirement. Inside analysis of the detail that it was proposed by developers of imprinters, monitors and scanners, it has an enormously wealthy area of data prerequisites for colorimetric alignment and range tables.

Hypothetically, TIFF could hold up images with numerous bands (up to $64 \mathrm{~K}$ bands), random quantity of bits for each pixel, information cubes, and manifold images per file, together by thumbnail sub-sampled images. Supported colour spaces include gray-scale, pseudo-colour (any size), RGB, YCbCr, CMYK, and CIELab. TIFF's foremost potency is that it is an extremely supple and stand-autonomous layout to be supported by several image dispensation applications. the most general restrictions of TIFF is the requirement of any provisions for storing vector graphics and text description, however this is not extremely threatening to OCR as most of the detection mechanisms effort with raster picture illustration.

The bitmap is a raster picture configure extensively employed in the Computers below operating systems. BMP pictures are stored in a machine free bitmap format that authorizes the operating system to reveal the bitmap on some group of exhibit machine. This identifies pixel colours in a figure independent of the system employed by a display to describe colour. Windows versions 3.0 and afterwards sustain Run Length Encoded (RLE) formats for compact bitmaps that utilize 4 or 8 bits for every pixel. Compression decreases the floppy and memory storage necessary proposed for a bitmap. Noticeably, this density approaches at the cost of the colour range and intensity, but this doesn't give a difficulty for bi-tonal or gray-scale supported OCR schemes. Bitmap records are mainly finely equivalent for the storage of actual humankind pictures; multifaceted images could be rasterized in combination with video, scanning, and photographic apparatus and stored in a bitmap format. BMP is a directly forward picture configure that is entirely appropriate for storing article pictures, but it isn't as complex and bendy as TIFF.

PCX be individual of the oldest raster formats obtainable PC's along with in the beginning recognized by Z-soft for its PC base Paintbrush software. Since it has been approximately for such a extended occasion, there are at the present lots of version of PCX. Generally software nowadays supports description 5 of the PCX arrangement. Adaptation 3 single supports 256 standards, but it does not permit for a tradition palette. This means that while you open an adaptation 3 PCX file, a benchmark VGA palette is old as a substitute. PCX retain all image in sequence (comparable to BMP) but it utilizes no compression, and hence could be memoryinefficient. 
The International Journal of Computational Science, Information Technology and Control Engineering

(IJCSITCE) Vol.4, No.1, January 2017

Joint Photographic Experts Group (JPEG) is the inventive given name of the board that invented this benchmark for lossy picture density that forfeits some picture superiority to significantly minimize the file dimension. JPEG was designed for compressing occupied-colour or gray-scale images of usual, existent-earth scene. It works fine on photograph, naturalistic artwork, as well as analogous fabric; not so well on inscription easy comics, or line drawings. JPEG compression exploits the reality so as to human eyes perceive small colour changes fewer precisely than little changes during clarity. Therefore, JPEG be proposed for compressing images so as to determination exist looked at through humans. Nevertheless, little faults initiated by JPEG compactness might be challenging for mechanism vision schemes; and specified it's fewer than ample concert on bi-tonal imagery, JPEG isn't a suggested picture file configure in OCR.

GIF stands for Graphic Interchange Format. It was admired in the 1980s the similar as a well prepared resources to transmit pictures crossways details networks. Through near the beginning 1990s, GIF was adopted as a result of the World Wide Web for its effectiveness and extensive knowledge. Today the irresistible majority of images on the Web are in GIF format. GIF files are partial to 8-bit colour palettes supporting refusal additional than 256 colours. The GIF format incorporates a compression system in the direction of remain folder sizes at a minimum. GIF works for pictures of manuscript and figures that are superior to actual world pictures. This crafts GIF a striking alternative for OCR schemes that effort with bi-level and gray-scale pictures.

PNG stands for Portable Network Graphics. This devise be intended to phase away the grown-up and simpler GIF format, and to afford a sensible substitute toward the greatly additional compound TIFF format. On behalf of the Web, PNG actually has three most important advantages above GIF: alpha channels (inconsistent intelligibility), gamma improvement (crossplatform manages of image brightness), in addition to two-dimensional interlacing (a technique of progressive exhibit).

As acts GIF, PNG supplies a lossless compactness, however acts an enhanced trade through it. Altered GIF, PNG supports simply a solitary image for each file. Nothing like TIFF, the PNG requirement is strict on implementation of supported format features, which translates into much better (than TIFF) representation file portability. Nevertheless, TIFF's group for fax firmness or else the JBIG setup is repeatedly again secluded enhanced than 1-bit gray-scale PNG for black and white pictures of manuscript or sketches.

These affluent varieties of picture formats could be vast to maintain in a distinct OCR method, and picture format translators frequently have to be utilized. Such translators might be accessible as

- Stand alone or open source service programs (for example: bmp to tiff)

- Ingredient of graphics libraries (for example: Image Magic)

- Ingredient of business picture procedure software (for example: Adobe Photo Shop)

Even if concerned with principally by digitally scanned manuscript articles, OCR method is presently encountering extended applicability to other media for instance digital picture, video, and the WWW. The primary task there remains the same: finding text in images; but the image format does not have to be fixed to black-and-white. Functioning in this tough domain, investigators practise the vital objective of structuring an OCR method that could identify any manuscript in any motionless or motioned photo programmed in any configuration. The subsequent subdivisions talk about picture configurations that are conventional for OCR and not so conventional for it. By evaluate every secluded alphabet with a database of predefined alphabets to identify it. 
The International Journal of Computational Science, Information Technology and Control Engineering (IJCSITCE) Vol.4, No.1, January 2017

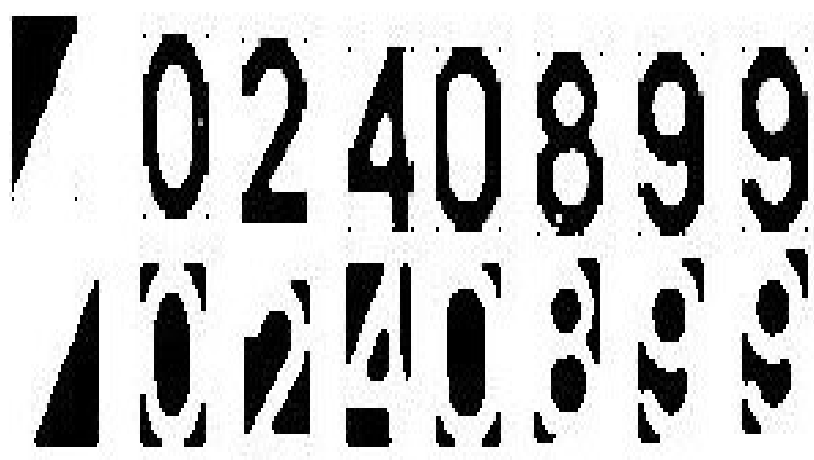

Fig.2. Segmented Characters

Segmented Characters consists of the following:

- Adjusting the color of the plate.

- Increasing the contrast.

- Remove all objects containing fewer pixels than expected in the alphabets.

- Filtering the obtained objects from noise.

- Isolating each alphabet.

The improvements in recognition of pattern has speed up currently caused by the lots of promising appliances which aren't merely tough, but moreover computationally further tough, such conspicuous inside OCR for instance, text categorization, Computer revelation, information Mining, alphabet detection, and Biometric validation [5]. The domain of OCR is suitable for fundamental element of article digital scanners, and is employed in lots of applications for instance postal procedure, handwriting identification, banking, safety (i.e. passport validation) and speech recognition. The examination in this domain has been continue for over half a century and the results have been surprising with victorious detection rates for written alphabets greater than $99 \%$, with noteworthy enhancements in act for handwritten feature recognition where detection rates have surpassed the $90 \%$.

A handwritten autograph is the concluding reaction to a compound cognitive and neuromuscular development which is the effect of the growing up and learning functions. This begins through upbringing with processions and doodles. Afterwards, immature brood initiate to employ communication in their acquire manner, while their motor manage is frequently not extremely perfect. Tracing assists both to improve their motor control and the learning of the shape along with spatial associations among substance, therefore create the spatial recollection or else cognitive map. Formerly this information is acquired and the motor skills begin to mature, it is likely in the direction of pick an ordered progression of aim points to execute flowing and effortless handwriting. By this phase, the human being is prepared toward describe with perform his or her own signature [17]. While signature description, which is the foundation of inter individual inconsistency, is scarcely customized, its implementation modifies constantly because of lengthy and small idiom features. The lengthy idiom roving is mostly caused by aging while small idiom changeability is caused by either psychosomatic causes or exterior features for instance different attitudes, scripting tools, signing surfaces, or other inexplicable causes. Modelling this variability's is a key issue in signature examination and it is delighted another way in numerous methodical domains. 


\section{LITERATURE SURVEY}

This segment presents several discussions regarding numerous OCR systems existing nowadays. In "[1]", proposed algorithm could be damaged as a kernel utilized for solitary alphabet finding within an entire OCR description method with no requirement for any compound mathematical operations. The highlights of this approach are that, it doesn't employ any databases or libraries of picture matrices to differentiate alphabets, but it has a special algorithm to differentiate alphabets in its position. The enthusiasm for the improvement of that algorithm was the straightforward actuality that English alphabets are permanent glyphs and they shall not be altered forever. Caused by this reality, procedure of non expected neural networks and vector based information education supply approximately perfect outcomes, but these are performing many outmoded work.

Furthermore, nearly all of the Optical Alphabet Detection process nowadays involves descriptions from high resolution scanners and cameras. Optical Alphabet Recognition technology currently could construct utilization of this progression in knowledge and believe techniques which were discarded appropriate to the requirement of in attendance day imaging skill. That algorithm is single such move towards. That algorithm has the improvement of speed, power, and recollection with area, while it does not comprise any instruction or knowledge mechanism and also since of lack of image database which various Optical Alphabet Detection technique need. As well, that algorithm is the primary in organism a manifold font Optical Alphabet Recognition and also a no preparation style Optical Alphabet Identification. To finish, the inhabited algorithm be able to willingly be old as a kernel within an absolute OCR description to be familiar with every alphabet after the segmentation with such adjustment operation to bring into line the alphabet parallel. The algorithm too gives a correctness of $100 \%$ on the at hand test set of alphabets for mainly of the fonts in all the three over mention font family at present. Potential version of this algorithm could be usual to take out additional type of description from the input alphabet image to development correctness and to afford support for additional alphabet sets.

In "[2]", a license number plate is considered as the exclusive acknowledgment of a medium, which builds the license number plate detection a crucial operation in intellectual transportation systems (ITS). Given that very many method associated to the LPR is inadequate to specific operational conditions, a multi filter base LPR structure for the protect localization and the alphabet identification is projected to resolve the issue. In the localization stage, chromatic and morphologic filters are cooperating through additional beneath flexible criterions to notice applicant plate region precisely. Plate description, such as the distance end to end-to-width fraction, the size of a quality, etc. are utilized in the temperament segmentation period. In the identification stage, a back propagation (BP) neural network is taught for the disposition detection. 800 images taken from an assortment of scene in dissimilar situation are old to assess the correctness of the anticipated structure. The investigational consequences illustrate that the absent velocity of localization is secure up to nothing, and the correctness of the plate localization and the identification is $98.4 \%$ as well as $93.8 \%$ respectively. Moreover, the overall accuracy of the multi-filter base structure is $93.1 \%$.

For locating license plates, separating and recognizing alphabets in a plate successfully, a multifilter based LPR framework is proposed in that paper. The main feature of that framework is the capability of combining multiple filters to ensure the missing rate is close to zero in localization with high flexibility. Specifically, filters with different specific methods could be add or changed based on the physical environment.

In "[3]", Writer provides a well-organized algorithm for habitual recognition of any license plate, through an emphasis on the Lebanese license plates wherever a few of their features have been 
The International Journal of Computational Science, Information Technology and Control Engineering (IJCSITCE) Vol.4, No.1, January 2017

exploited well to decrease the identification mistakes. The planned algorithm has been implemented through the illustration Processing Toolbox during MATLAB R2013b (8.2.0.701). Their experiments exhibit to the detection inaccuracies have been focused well upon developing the actuality that the Lebanese license shields are printed in two designs. Also, their method has an alternative to profit from the corporation of the license shields in the front together with the back end of the car to develop the appearance.

In "[4]", author presented alphabetistic extraction technique in usual scene images. Along with expand alphabet recognition scheme using alphabetistic extraction technique in natural scene images. A graph matching method utilized structural alphabetistic of alphabet. It is recognition technique to facilitate it consider relative of location and structural relation. It is robust technique to change of font or rotation. In organize to proof it; they experienced two cases that training font and test font are similar case or distinction case.

In "[5]", they evaluate a fresh alphabet recognition technique of certify plate numeral based on similar BP neural networks. This enhanced the correctness of the identification scheme that aims to understand writing repeatedly the Chinese license plate. In the planned method, the quality is binarized with the sound is eliminate in the pre-processing stage, then the alphabet alphabetistic is extract with frame by means of the alphabet is normalize to size $8 * 16$ pixels. As a final point, the alphabet attribute is place addicted to the similar neural network and the alphabet is documented. The anticipated technique in alphabet recognition is effectual, and hopeful grades have been obtain in experiment on Chinese certify plates.

They compare the alphabet gratitude presentation using the parallel neural networks with other methods. They gave the compare of the alphabet recognition rate and recognition time among three methods in which method 1 represents their proposed method, method 2 is the method of using simple BP neural networks, and method 3 is the method of using template matching. They utilized 400 number-plates to test their algorithms. The experimental results shown that this process could improve more than $8-10 \%$ correct rate of alphabet recognition comparing with method 2, while 6-8\% compare with method 3 . The timing of recognition using their method is close to method 2 , and is less than method 3 . In addition, using method 1 , the probability which two alphabets and more than two alphabets in the same number-plate are all mistake recognition is less than $4 \%$, that performance is better than that using other two methods. In most case, the time of all seven alphabets recognition is close to 0.01 second, and the recognition rate of these alphabets is about $98.88 \%$.

In "[6]", they tried to know printed and handwritten alphabets with projecting them on dissimilar sized grids $(5 \times 7,7 \times 11$, and $9 \times 13)$. The consequences showed that the accuracy of the quality recognition depends on the resolution of the alphabet projection. As well, they realized that not each inscription approach could be expected with the similar network through the identical accuracy. This shows that the diversity of individual handwriting habits could't be completely enclosed through single neural network.

In "[7]", they analysed programmed learning systems utilized to extract functional information from musical scripts, participate a major role in optical music recognition. Visual music acknowledgment or OMR has been extensively utilized to take out the melodious notations and understanding as of old scripts and thus encloses lot of significance in retrieving chronological data. The field of pattern recognition along with knowledge illustration has to be symmetrically utilized for music notation recognition making it a demanding problem. Nearly every one of the methods utilized for OMR acknowledgment and extraction like HMM's, Neural etc mentioned in literature have mistakes which necessitate being operators to exist rectified. The paper planned fresh automatic music recognition systems which know how to be efficiently utilized to recognize 
The International Journal of Computational Science, Information Technology and Control Engineering

(IJCSITCE) Vol.4, No.1, January 2017

sheet or printed scores into a playable platform under the unlike conditions of blur, explanation variations, noise etc. The manuscript utilized an improvement supported threshold GA based preprocessing methodology for bimodal images additional compares through the Sable filtered and usual GA outputs for the similar. The attitude makes utilize of an enhanced image obtained by histogram equalization followed by image segmentation using a definite threshold. The threshold could be obtained using inherited algorithms. GA based segmentation method is codified as an optimization problem utilized professionally to search maxima and minima from the histogram of the image to gain the threshold for segmentation. The scheme described is able of extracting usual images as well as images through noise, images as of older scripts.

In "[8]" They studied the problem of improving the efficiency of In common; an ALPR system consists of four dispensation stage. In the picture gaining phase, a number of points have to be consider when choosing the ALPR scheme camera, such as the camera motion and the shutter speed.

In "[9]" In that work, they have utilized a license plate dataset consisting of 141 images. Note that the dataset is no homogeneous, as it contains images of various resolutions, aspect ratios, background contents, plate sizes, illumination conditions, camera angles, tilts, pans, etc. Most of the images utilized were captured in Ontario -Canada and hence, the dataset contains mostly Ontario plates. To normalize the input images, an aspect ratio of 1.33 is maintained and all of the images are scaled to $1024 \times 768$ pixels. For images with different aspect ratio, either the height or the width is scaled to keep the aspect ratio fixed.

In "[10]", that paper presents, there is as well a number of achievement by handwriting identification, mainly for inaccessible hand printed typescript and words. For example, in the online case, the recently introduced PDAs haw sensible value. Correspondingly, some online signature confirmation system has been market over the last few years and instructional tools to help children study to write are starting to emerge. Generally offline victories have arrive in inhibited fields, for instance postal addresses, bank cheques, and study structures. The assessment of authorize with complex layouts, credit of degraded printed text, and the detection of running handwriting maintain to stay mainly in the investigate arena.

In "[11]" visual quality detection refers toward a procedure whereby printed credentials be distorted into ASCII records intended for the reason of compact storage, editing, quick recovery, along with other file manipulations during the exploit of a processor. The appreciation phase of an OCR procedure is prepared hard by additional noise, image deformation, in addition to the variety of alphabet typefaces, size, with fonts to a text could contain. During to learn a neural network come near is introduce to execute elevated correctness detection on multi-dimension along with multi-basis typescript; a fresh centred-hesitant preparation procedure through a short noise-sensitivity normalization method is old to attain elevated correctness grades. The revision consists of two parts. The primary measurement focuses scheduled simply size and only font typescript, and a two-covered neural system is skilled to be familiar through the occupied deposit of 94 ASCII alphabet images in 12-pt dispatch rider foundation. The next part trades accuracy for supplementary font and size potential, and a better two-encrusted neural system is taught to be familiar with the complete set of 94 ASCII quality descriptions for every point sizes beginning $\mathrm{S}$ to 32 along with for 12 frequently utilized fonts. The arrangement of these two systems is estimated founded on a evidence of supplementary than discrete million alphabet depictions from the harsh dataset.

In "[12]", that papers present a straightforward, well-organized, and fewer costly approaches to create OCR for interpretation some manuscript that has fix font size and method or handwritten style. To full capability and less calculation rates, OCR in along the purpose of manuscript 
The International Journal of Computational Science, Information Technology and Control Engineering (IJCSITCE) Vol.4, No.1, January 2017

employs database to identify English alphabets which constructs this OCR system extremely straightforward to handle. That paper tells about OCR system intended for offline handwritten alphabet detection. The system has the ability to yield excellent results. Pre-dispensation technique utilized in document images as a first step in alphabet acknowledgment systems was obtainable. The feature extraction step of optical alphabet detection is the generally important. It could be utilized with existing OCR methods, especially for English text. This scheme suggests a higher boundary through having an improvement specifically its extensibility, specifically while it is organized to interpret a pre described set of article configures, at present English articles; it could be organized to identify original categories.

The artificial invention of standing handwritten signature base on vehicle correspondence hypothesis has been freshly planned for biometric application. Vehicle correspondences divide the human handwriting act interested in an effectors dependent cognitive level and an effectors self-governing vehicle altitude. The primary stage has been optional by others as an engram, generate during a spatial network, and the flash has been emulate with kinematic filters. Their manuscript planned an expansion of this method in which they produce energetic information and make available an incorporated all-inclusive synthesizer for both stationary and active autograph amalgamation. The dynamics are intended by lognormal example of the 8-linked constant signature curve, which include, as an innovation, the pen-ups. The counterfeit productions imitate a signature by extract the mainly perceptually applicable point of the specified authentic signature and interpolating them. The ability to manufacture together stationary as well as energetic signatures with an exceptional representation is evaluated according to its aptitude to get utilized to the stationary and energetic signature inter- and intra-individual unpredictability. Our extremely promising marks propose the opportunity of by the synthesizer in dissimilar area further than the invention of unrestricted database for biometric instruction [17].

\section{PROBLEM DESCRIPTION IN OCR}

Being a glowing industrial technological field, optical alphabet detection is generally utilize today for projects of a variety of scales, from infrequent document scanning to creation of massive digital document archives. Yet it remainders an area of active technical research and creative engineering. We might categorize the following most important investigation developments in the recent OCR domain. Flexible OCR endeavours at vigorous usage of an extensive variety of printed article imagery by addressing:

- Multi alphabets and multi language identification

- Omni alphabets manuscripts

- Involuntary article distribution

- Numerical document identification

Handwriting identification is a growing OCR method with the aim of to be enormously vigorous and flexible. Normally, it stays a dynamically investigated open difficulty to facilitate solution to a definite quantity for several distinct utilizations, for instance:

- Identification of given printed manuscript in documents

- Handwriting identification in individual cheques

- Postal packet and package address scanners

- OCR in moveable and handheld appliances

Script picture enhancement entails determining and implementing suitable picture filters to the origin article picture to facilitate the specified OCR mechanism enhanced to identify alphabets 
The International Journal of Computational Science, Information Technology and Control Engineering (IJCSITCE) Vol.4, No.1, January 2017

and phrases. Intellectual post procedure is of immense significance for getting better the OCR identification exactness and for constructing vigorous information retrieval (IR) schemes that employ elegant indexing and estimated series matching methods for storage and recovery of deafening OCR outcome manuscripts. OCR in multimedia is a motivating improvement that prepares method of optical alphabet identification in the media other than written papers, e.g. photo, video, and the Internet.

\section{A. Fundamentals of OCR system}

Nowadays wide range of systems to optical alphabet recognition is available. Several of them involve in pre distribution into identification of articles, and several of them don't, however we might securely suppose that some OCR algorithm would detachments these two fundamental elements:

- A alphabetistic excerptor

- A categorizer

Given an item picture, the characteristic extractors derive the characteristic (descriptors) that the item possesses. The traditional features are then previous as donation to the categorizer that resolves the identified article equivalent to the examined characteristic. The classifier is also expected to give a confidence level number that tell show certain the classifier is about the recognized item. Let us briefly describe some of the classical optical alphabet detection methods. Pattern corresponding is solitary of the mainly ordinary and basic classification methods. It is also known as matrix matching. A set of every alphabet picture models, patterns, is composed and identified a priori. The characteristic excerptor employs individual image pixels as features. Alphabet categorization is performing with compare a contribution nature representation against the template array. Every evaluation marks in a comparison compute (given by a known "distance" function) connecting the contribution disposition and the pattern. The similarity increases while a pixel in the experiential alphabet is indistinguishable to the identical pixel in the pattern image (match). When the corresponding pixels differ (mismatch), the measure of similarity may decrease. Accordingly, the alphabet's characteristic are allotted to be the single with the mainly similar pattern.

Constitutional classification systems occupy constitutional descriptions and conclusion regulations to categorize alphabets. Constitutional definition perhaps discrete in circumstances of tendency strokes, quality hole, or previous alphabet attribute such as corners and concavities. For example, the dispatch "P" might be describe as a "vertical stroke with a curved stroke attach on the higher accurate elevation making up a hole". To classify the alphabet, the input alphabet structural description is extract and a regulation-based organization is functional to compute the alphabet's class. Remind it the above discussed methods are illustrated in their canonical structure; however they have lots of procedural deviations and fusion methods. For example, pattern matching doesn't have to be pixel based, e.g. one might equivalent wavelet converts of pictures and patterns. Constitutional categorization doesn't constantly rely on verdict regulations, e.g. one might occupy an adjoining acquaintance categorizer through a suitable metric in the characteristic space. Many modern OCR systems are base on arithmetical formalisms that decrease some measure of misclassification. Such alphabet recognizers possibly will develop pixel-base description or structural features. Let us mention some of them: Discriminate function classifiers utilize hyper surfaces in multi-dimensional feature spaces to separate the feature explanation of typescript as of dissimilar semantic programme. Such classifiers aim at reducing the mean-squared classification mistake. 
The International Journal of Computational Science, Information Technology and Control Engineering (IJCSITCE) Vol.4, No.1, January 2017

\section{B. Optical Alphabet Identification Inaccuracy through their result on usual speech Processing}

A short summary of every stage in addition to its potential problem areas is listed in Table 1 . The interactions between mistakes that arise throughout OCR and later stages could be complex. A number of common scenarios are depicted in Fig. 3. It is straightforward to visualize a solitary mistake proliferating the entire way to the channel and recall an equivalent mistake at all of the afterwards stages in the procedure.
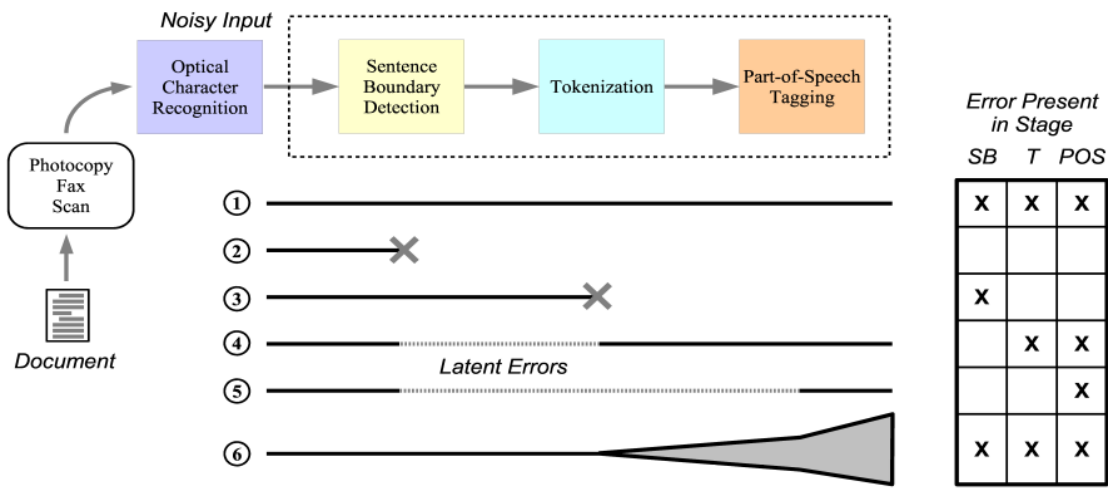

Fig.3. Propagation of OCR mistakes through NLP stages [18]

Mistakes are unavoidable [18] in complex processor image application such as visual quality detection; with the sound induce by these mistakes present a serious face up to do upstream process that efforts to create make utilize of such information.

Table.1 Processing steps of manuscript

\begin{tabular}{|c|c|c|}
\hline Processing step & Intended occupation & Potential difficulties \\
\hline OCR & $\begin{array}{c}\text { Copy contribution bitmap into } \\
\text { determined manuscript }\end{array}$ & $\begin{array}{c}\text { Current OCR is "brittle;" } \\
\text { mistakes made early-on } \\
\text { propagate to later stages. }\end{array}$ \\
\hline $\begin{array}{c}\text { Sentence } \\
\text { boundary } \\
\text { reorganization }\end{array}$ & $\begin{array}{c}\text { Break input into sentence-sized units, } \\
\text { one per text line. }\end{array}$ & $\begin{array}{c}\text { Absent or bogus phrase } \\
\text { limitations because of OCR } \\
\text { mistakes on punctuation. }\end{array}$ \\
\hline Tokenization & $\begin{array}{c}\text { Crack all phrase into word } \\
\text { tokens enclosed by blank space. }\end{array}$ & $\begin{array}{c}\text { Absent or bogus tokens } \\
\text { because of OCR mistakes on } \\
\text { blank space and punctuation. }\end{array}$ \\
\hline $\begin{array}{c}\text { Division of } \\
\text { language } \\
\text { classification }\end{array}$ & $\begin{array}{c}\text { Obtains tokenized manuscript and } \\
\text { envoys label to every token } \\
\text { representative its part of speech. }\end{array}$ & $\begin{array}{c}\text { Awful PoS marks because of } \\
\text { failed tokenization or OCR } \\
\text { mistakes that change } \\
\text { orthographies. }\end{array}$ \\
\hline
\end{tabular}

\section{Complications in optical alphabet recognition}

Alphabets misclassifications shoot from two major bases: poor superiority identification part pictures and insufficient unfair capability of the categoriser. There are lots of issues that donate to deafening, tough to distinguish article imagery: 
The International Journal of Computational Science, Information Technology and Control Engineering

(IJCSITCE) Vol.4, No.1, January 2017

- deprived novel article superiority

- deafening, small resolution, multi invention picture scanning

- wrong or inadequate symbol pre indulgence

- deprived segmentation into identification articles

At the other side, the alphabet recognition procedure itself might require an appropriate reply on the specified alphabet set, thus ensuing in categorization mistakes. This sort of mistakes might be complex to treat because of an inadequate instruction set or inadequate learn abilities of the categoriser. Typical identification rates for system written font might achieve more than $99 \%$ other than handwritten disposition detection rates are invariably lower because every person writes differently. This arbitrary behave frequently manifests itself in a superior alphabet difference in the characteristic space foremost to superior misclassification rates. A general instance of a "complicated" alphabet is the letter "O" simply mystified with the number " 0 ". An additional superior instance might be the letter "l" mystified with the number "1" or incorrect for a deafening picture of the letter "I". The peak level of their catalogue of mistake origins consists of

- Imaging faults because of weighty/light print drift stains, arced baselines, etc.

- parallel symbols as revealed above

- Punctuation because of commas and phases, citation spots, exceptional symbols, etc.

- improved picture processing found on additional realistic modeling of the printing, and scanning processes

- Adaptive alphabet categorization by fine alteration the categorizer to the existing manuscript's font

\section{CONCLuSion}

We present a review work of Performance examination of optical alphabet identification. In study, we analyzed the theoretical and mathematical model of the most challenging problem in the scope of optical alphabet identification is trade through scale, translate and rotate quality in Optical alphabet detection. The probable deployments of the OCR methods are also studied, the precision and identification isn't adequate for realistic deployment. It may require significant improvement. In future we will be trying to implementing an OCR system using not only a mobile phone for all tasks. There are numerous efforts that still require being finished. Several extra OCR methods and algorithm will be integrated in this examine and widespread experiments require to be completed with superior amount of pictures.

\section{REFERENCES}

[1] Sushruth Shastry, Gunasheela G, Thejus Dutt, Vinay D S and Sudhir Rao Rupanagudi, “ $i$ ” - $A$ novel algorithm for Optical Alphabet Recognition (OCR). 978-1-4673-5090-7/13/\$31.00 @2013 IEEE.

[2] Lulu Zhang, Xingmin Shi, Yingjie Xia, Kuang Mao, "A Multi-filter Based License Plate Localization and Recognition Framework”. 978-1-4673-4714-3/13/\$31.00 @2013 IEEE.

[3] Ibrahim El Khatib, Yousef Samir-Mohamad Omar, and Ali Al Ghouwayel, "AN EFFICIENT ALGORITHM FOR AUTOMATIC RECOGNITION OF THE LEBANESE CAR LICENSE PLATE. ISBN: 978-1-4799-5680-7/15/\$31.00 @2015 IEEE.

[4] Jieun Kim, and Ho-sub Yoon "Graph Matching Method for Alphabet Recognition in Natural Scene Images. 978-1-4244-8956-5/11/\$26.00 @2011 IEEE

[5] Feng Yanga, and Fan Yangb, “Alphabet Recognition Using Parallel BP Neural Network”. 978-14244-1724-7/08/\$25.00@2008IEEE. 
The International Journal of Computational Science, Information Technology and Control Engineering (IJCSITCE) Vol.4, No.1, January 2017

[6] Rókus Arnold, and Póth Miklós "Alphabet Recognition Using Neural Networks. 11th IEEE International Symposium on Computational Intelligence and Informatics • 18-20 November, 2010 - Budapest, Hungary.

[7] Amarjot Singh, Ketan Bacchuwar, Akash Choubey, and Devinder Kumar , "An OMR Based Automatic Music Player". 978-1-61284-840-2/11/\$26.00 @2011 IEEE.

[8] Shan Du, Member, IEEE, Mahmoud Ibrahim, Mohamed Shehata, Senior Member, IEEE, and Wael Badawy, Senior Member, IEEE "Automatic License Plate Recognition (ALPR):A State-ofthe-Art Review, IEEE TRANSACTIONS ON CIRCUITS AND SYSTEMS FOR VIDEO TECHNOLOGY, VOL. 23, NO. 2, FEBRUARY 2013.

[9] Imran Shafiq Ahmad, Boubakeur Boufama, Pejman Habashi, William Anderson and Tarik Elamsy, "Automatic License Plate Recognition:

[10]A Comparative Study". 2015 IEEE International Symposium on Signal Processing and Information Technology (ISSPIT).

[11] Rejean Plamondon, Fellow, IEEE, and Sargur N. Srihari, Fellow, IEEE, “On-Line and Off-Line Handwriting Recognition:A Comprehensive Survey 1EEE TRANSACTIONS ON PATTERN ANALYSIS AND MACHINE INTELLIGENCE. VOL. 22, NO. 1. JANUARY 2000.

[12]Hadar I. Avi-Itzhak, Thanh A. Diep, and Harry Garland, "High Accuracy Optical Alphabet Recognition Using Neural Networks with Centroid Dithering IEEE TRANSACTIONS ON PATTERN ANALYSIS AND MACHINE INTELLIGENCE, VOL. 17, NO. 2, FEBRUARY 1995.

[13] Shalin A. Chopra, Amit A. Ghadge, Onkar A. Padwa, Karan S. Punjabi, Prof. Gandhali S. Gurjar, "Optical Alphabet Recognition", International Journal of Advanced Research in Computer and Communication Engineering Vol. 3, Issue 1, January 2014.

[14]C. Nelson Kennedy Babu, Member IEEE and Krishnan Nallaperumal, Senior Member IEEE, " $A$ License Plate Localization using Morphology and Recognition”, 978-1-4244-2746-8/08/\$25.00 (C)2008 IEEE.

[15] S. N. Nawaz, M. Sarfraz, A. Zidouri, and W. G. AI-Khatib, "AN APPROACH TO OFFLINE ARABIC ALPHABET RECOGNITION USING NEURAL NETWORKS”, 0-7803-8163-7/03/\$17.00 02003 IEEE.

[16] Aparna.A, and Prof. I.Muthumani, "Optical Alphabet Recognition for Handwritten Cursive English alphabets", International Journal of Computer Science and Information Technologies, Vol. 5 (1), 2014, 847-848.

[17] Jacob George, Varghese George, A. Deepan, Joshua Ninan Valson, "Optical Alphabet Recognition Based Hand-Held Device for Printed Text to Braille Conversion 978-1-4244-925 3-4 /11/\$26.00 (C)2011 IEEE.

[18] Miguel A. Ferrer, Moises Diaz, Cristina Carmona-Duarte and Aythami Morales, “A Behavioral Handwriting Model for Static and Dynamic Signature Synthesis: IEEE TRANSACTIONS ON PATTERN ANALYSIS AND MACHINE INTELLIGENCE 2016. 